\title{
Precision spraying: from map to sprayer control using model-checking
}

\author{
Rim Saddem $^{1,2}$, Olivier Naud ${ }^{1}$, Paul Cazenave ${ }^{1}$, Karen Godary Dejean ${ }^{2}$, Didier Crestani ${ }^{2}$
}

\section{N F O}

Received 17 Jul 2017

Accepted 15 Nov 2017

Available on-line 21 Nov 2017

Responsible Editor: M. Herdon

\section{Keywords:}

Precision agriculture, timed automata, optimization, grapevine, LiDAR data.

\begin{abstract}
$\underline{\text { A B S T R A C T }}$
Precision agriculture is a set of methods based on spatial and timed information which aims at making agricultural operations specific to local crop needs. This study was conducted to contribute to precision spraying for trellised perennial crops such as grapevine with a new method called Automata Modelling for Precision Spraying (AMPS). AMPS is based on the timed automata formalism and the UppAal-CORA model-checking tool with cost optimisation. It takes 2D ground-based LiDAR data of the canopy as input and computes a command sequence for the sprayer that is optimized for a cost criterion. AMPS was tested on LiDAR data from a vine row and with a hypothetical sprayer model based on pneumatic technology and individual command for each spout. The chosen cost criterion was the amount of product sprayed on the row. It was demonstrated on the example that a significant proportion of phytosanitary product can be saved. It was also shown that it is necessary to take into account spout control dynamics, because the savings are inferior to the ideal case in which spout response would be instantaneous.
\end{abstract}

\section{Introduction}

Precision agriculture has for origin the development, in recent decades, of localization and sensing technologies by which site specific management can be implemented in the field, in order to optimize a given criterion, e.g. economical or environmental benefit (Berk \& al. 2016). Most recent technologies like multispectral imagery and laser scanning, whether airborne or from the ground, allow the acquisition of crop data with a large spatial resolution (Rosell et al., 2009 ; Hall et al., 2003). On perennial crops such as grapevine, sensors can be used to quantify and map the density of vegetation in the canopy. This specific and spatial information is an opportunity for automation specialists to develop innovative and efficient methods for accurate spraying of phytosanitary products. Such accurate spraying may contribute to reduce the quantities of chemicals sprayed while maintaining yield and quality.

The research presented here is a contribution to the development of command methods for sprayers in order to optimize spraying according to the vegetation, in the case of trellised crops such as grapevine. In particular, considering the control dynamics of a sprayer, we investigate the possibility to apply a previously determined map of pesticide dose and spraying configurations. Our contribution, called Automata Modelling for Precision Spraying (AMPS), is based on the formalism of Timed Automata (TA) and on the UppAal-CORA model-checking tool. Timed Automata, introduced by (Alur and Dill, 1994), is a formalism used for the modelling of real-time discrete systems. This type of representation, which is widely used for the specification of industrial automation, has also been developed in research for agriculture or ecosystem management (Hélias et al., 2008; Largouet et al., 2012; Scopélitis et al., 2007). UppAal is a tool designed to validate, through properties verification, systems that can be modelled as networks of TA. The language used in UppAal extends the formalism of TA by adding integer variables, structured data types, user defined functions, and channel synchronization (Larsen et al., 1997). UppAal-CORA brings a cost optimisation feature on top of model-checking (Berhmann et al, 2005).

\footnotetext{
${ }^{1}$ Irstea, UMR ITAP, 361 rue Jean-François Breton, BP 5095, F-34196 Montpellier, France

${ }^{2}$ Laboratoire d'Informatique Robotique et Microélectronique de Montpellier (LIRMM), UMR 5506, Université de Montpellier, 161 rue Ada, 34095 Montpellier Cedex 5, France.
} 
Several authors such as in (Gil et al., 2007) and (Pai et al., 2009) have developed automation methods for sprayers. The evaluation is usually empirical: once the control has been designed and implemented, it is tested and performances are analysed. On the contrary, the AMPS method is an attempt to estimate performances to expect before developing an automated sprayer. The study is based on the hypothesis that a pneumatic type sprayer would be equipped with individual control of the spouts. Yet, AMPS was developed to offer adaptability to other spraying technologies. AMPS considers two criteria: guarantee of sufficient crop protection on each vine plant, and overall reduction of quantities at plot scale. The outline of the paper is as follows. Section 2 describes the characteristics of the sprayer used in the work. Section 3 introduces Automata Modelling for Precision Spraying method. In Section 4, AMPS is applied to real vine LiDAR data (Light Detection And Ranging) and the control sequence obtained is compared, for the same vine case, with what would be obtained with results observed with an unautomated sprayer.

\section{Description of sprayer}

The work is based on a hypothetical sprayer derived from classic pneumatic spraying technology for grapevine and orchards. For the sake of simplicity, only "hand" shaped spout types are considered here. A classic grapevine sprayer has two Hands: one Low (LH) and one High (HH). Usually, both are used at the same time. To address precision agriculture, we suppose that our hypothetical sprayer can switch on/off these hands independently and has a third Central Hand $(\mathrm{CH})$, which can cover approximately same crop height as LH and HH used together (figure 1). Each hand has the same flow rate, which is half the nominal flow rate (the nominal flow is the one that is obtained when LH and $\mathrm{HH}$ are used simultaneously).

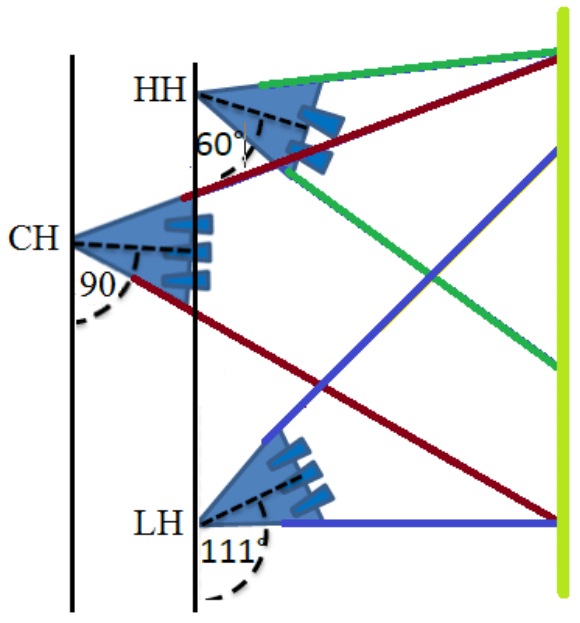

Figure 1. Hypothetic automated pneumatic sprayer: layout of spouts (3 hand shaped spouts)

\section{Description of the Automata Modelling for Precision Spraying method}

This section presents the AMPS (Automata Modelling for Precision Spraying) method that we have developed in order to support control design and address precision agriculture issues. This method takes 2D ground-based LiDAR data of the canopy as input and computes, for a given sprayer with individual control of spouts, a command sequence optimized for a cost criterion while ensuring a sufficient protection on each vine plant. For the sake of simplicity and genericity, we will call here after in the model each spout a "nozzle". AMPS is based on 3 steps: (1) define a function "Precision Sprayer Mapping (PSM)" that maps each vegetation state to the desired spraying configuration for this state. (2) apply this mapping to localised vegetation state data, (3) compute and verify spraying control using a model based on Timed Automata and on the UppAal-CORA model-checking tool, which has an optimisation feature (Behrmann et al., 2005). In the following, each step is detailed. 


\subsection{Step 1: Definition of the Precision Sprayer Mapping}

The grapevine crop is organized in rows, and each row is considered as a list of successive crop blocks of various lengths. Each crop block is considered homogeneous in its characteristics. The height of a crop block is divided into 3 horizontal sections: Low $(\mathrm{L})$ for the canopy height between $\mathrm{h}_{1}$ and $h_{2}$, High $(H)$ for the canopy higher than $h_{3}$ and Middle (M) for the canopy height, between $h_{2}$ and $\mathrm{h}_{3}$. The number of LiDAR beam interceptions in each section within a block is supposed representative of foliage quantity and canopy porosity. Step1 of AMPS consists essentially in the static definition of a function, the Precision Sprayer Mapping (PSM) which takes as input abstract qualitative values derived from LiDAR raw data. For a given section L, M, H, 3 values can be considered: 0 is for no vegetation, 1 is for some (a little of) vegetation, 2 is for a lot of vegetation. These values are obtained from raw 2D LiDAR data according to thresholds. In this paper, thresholds were calculated with reference to statistics for each vertical section and the total height of one vine row. The threshold to discriminate between 1 and 2 was chosen as the median of the number of LiDAR beam interceptions in the considered horizontal section. The thresholds for abstract value 0 were chosen specifically for each horizontal section: exactly 0 interception for $\mathrm{L}$ section, below 30 LiDAR interceptions for a $10 \mathrm{~cm}$ wide scan slice for M section, and below $15 \mathrm{LiDAR}$ interceptions for a $10 \mathrm{~cm}$ wide scan slice for $\mathrm{H}$ section. For the whole crop height $\mathrm{T}$ (Total $\mathrm{T}=\mathrm{L}+\mathrm{M}+\mathrm{H}$ ), only values 1 and 2 are considered, because the decision to consider a crop block as "missing vegetation" is taken with regards to $M$ section (see yellow box in Table 1 ). $h_{1}, h_{2}$ and $h_{3}$ were respectively set to $0.3,0.6$ and $1.2 \mathrm{~m}$.

The PSM maps spraying configurations, i.e. the discrete set of commands that could be applied to the sprayer, to each value of the quadruplet $(\mathrm{T}, \mathrm{H}, \mathrm{M}, \mathrm{L})$. This mapping can be defined using expert knowledge. In this study, the PSM was produced using expertise based on testing actual sprayers on an artificial vine bench (Naud et al, 2014). An extract of PSM is described in table1 showing 24 of the $2 \times 3 \times 3 \times 3$ possible combinations of $(\mathrm{T}, \mathrm{H}, \mathrm{M}, \mathrm{L})$.

Table 1. Example of PSM information

\begin{tabular}{|c|c|c|c|c|c|c|c|c|} 
T-H-M-L & $\mathrm{C}_{\text {best }}$ & $\mathrm{C}_{\text {alt }}$ & T-H-M-L & $\mathrm{C}_{\text {best }}$ & $\mathrm{C}_{\text {alt }}$ & T-H-M-L & $\mathrm{C}_{\text {best }}$ & $\mathrm{C}_{\text {alt }}$ \\
\hline $\mathbf{x x 0 x}$ & -- & -- & 1110 & $\mathrm{HH}$ & $\mathrm{CH}$ & 2012 & LH & LH\&CH \\
\hline $\mathbf{1 0 1 0}$ & LH & HH & 1111 & $\mathrm{CH}$ & $\mathrm{CH}$ & 2021 & LH\&CH & LH\&HH \\
\hline 1011 & $\mathrm{LH}$ & $\mathrm{CH}$ & 1112 & LH\&CH & $\mathrm{CH}$ & 2022 & LH\&CH & LH\&HH \\
\hline 1012 & LH & LH & 1121 & $\mathrm{CH}$ & LH\&HH & 2112 & LH\&CH & LH\&CH \\
\hline 1021 & LH & $\mathrm{CH}$ & 1122 & $\mathrm{CH} \& H H$ & LH\&CH & 2121 & LH\&HH & LH\&HH \\
\hline 1022 & LH\&CH & LH\&CH & 1211 & $\mathrm{CH}$ & $\mathrm{CH} \& H H$ & 2122 & LH\&CH & LH\&CH \\
1212 & LH\&HH & LH\&CH & 2221 & CH\&HH & CH\&HH & 2211 & CH\&HH & CH\&HH \\
\hline 1221 & CH & CH\&HH & 2222 & LH\&HH & LH\&HH & 2212 & LH\&HH & LH\&CH \\
\hline
\end{tabular}

Two commands guaranteeing an effective crop protection were assigned to each vegetation block: the preferred command $\left(\mathrm{C}_{\text {best }}\right)$ which sprays the best dose (neither too high nor too low) with the most appropriate spraying configuration and the alternative command $\left(\mathrm{C}_{\text {alt }}\right)$ which provides a sufficient, yet possibly higher than necessary, local dose. When no satisfactory alternative can be defined, $\mathrm{C}_{\text {alt }}=\mathrm{C}_{\text {best }}$. 7 spraying configurations were considered (naming based on the spouts used): LH, CH, LH\&CH (which means that Low and Central hand must be used together), HH, LH\&HH, CH\&HH and -- for hands all switched off (missing vegetation: yellow boxes in Table 1). For example, if a vegetation block has the characteristics T-H-M-L $=(1010)$ (orange boxes in Table 1$)$ then $\mathrm{C}_{\text {best }}=\mathrm{LH}$ and $\mathrm{C}_{\text {alt }}=\mathrm{HH}$. In this case, $\mathrm{CH}$ command needs to be avoided because products would be wasted on top and on

Rim Saddem, Olivier Naud, Paul Cazenave, Karen Godary Dejean, Didier Crestani: Precision spraying: from map to sprayer control using model-checking 
bottom of vegetation. In the figure 1, it can be seen that High and Low Hands have an angle which makes LH spray on both $\mathrm{L}$ and $\mathrm{M}$ sections, and $\mathrm{HH}$ spray on both $\mathrm{H}$ and $\mathrm{M}$ sections. The results from tests done on the artificial bench indicate that $\mathrm{LH}$ is the best command, and $\mathrm{HH}$ is an alternative. The need for having two commands instead of only $\mathrm{C}_{\text {best }}$ results from nozzles response time. Choosing a unique local and ideal spraying configuration command for each block may not provide the optimal spraying on the whole field due to transition times in opening and closing nozzles. Indeed, for avoiding insufficient spraying, when spraying configuration is changed, any nozzle to be set ON needs to be activated before any nozzle to be set OFF is deactivated. This can cause to spray more when spraying configuration is changed too frequently. This effect of nozzles dynamics is precisely why a computing framework is needed for a better control design. It is important to outline that in this application of model-checking with optimisation, spraying enough product on each vine plant means production safety (vine production point of view) and that quantities sprayed overall on the whole vine plot should be optimised (environmental point of view).

\subsection{Step 2: Description of the Vegetation Block Definition-algorithm}

The step 2 (figure 2) consists in the processing of real LiDAR data to obtain $\mathrm{C}_{\text {best }}, \mathrm{C}_{\text {alt }}$ and the duration of each vegetation block for the studied row, considering the PSM detailed in step 1 . These LiDAR data consist in the scan of vine rows from a 2D LiDAR mounted on a tractor drived in vine inter-rows such as presented in (Bastianelli et al. 2017). The tractor speed was $5 \mathrm{~km} . \mathrm{h}^{-1}$. The type of LiDAR used in this experiment was LMS 100 . The data acquisition frequency was $50 \mathrm{~Hz}$, the angular scan range was $270^{\circ}$ with a $0.5^{\circ}$ resolution set up so as to scan vegetation on both sides as well as the ground. The outline of step 2 algorithm for a vine row is as follows. At first, the row is sampled in 10 $\mathrm{cm}$ vegetation slices (1). For each slice and each horizontal section within a slice, the number of LiDAR beam interceptions is counted (2). The criterion for assigning "missing vegetation" to a slice is, according to PSM, T-H-M-L = xx0x (3). Then, adjacent missing vegetation slices are grouped together into a single block (4). The resolution of $10 \mathrm{~cm}$ was chosen for the method in order to detect missing vegetation with precision and be able to open and close nozzles at right timings in this case. But it is not possible to consider such thin slices for control if they are not assembled, because at 1.4 m. $\mathrm{s}^{-1}$ speed (approx. $5 \mathrm{~km} . \mathrm{h}^{-1}$ desired speed for sprayer), $10 \mathrm{~cm}$ represent $0.07 \mathrm{~s}$ duration while opening or closing nozzles requires $0.2 \mathrm{~s}$. Then, the vegetation between missing vegetation blocs is divided into blocks of minimum length $0.5 \mathrm{~m}$. A new counting of LiDAR interceptions is made for these blocks and the thresholding described in previous section is applied to obtain the T-H-M-L values (5). Adjacent vegetation blocks with similar abstract values are grouped into a single block (6). For each block, $\mathrm{C}_{\text {best }}$ and $\mathrm{C}_{\text {alt }}$ are computed using the PSM function (7). Finally, considering a fixed sprayer velocity along the row, positions and distances (lengths of blocks) are converted to time points and durations which are used in the model depicted in step 3.

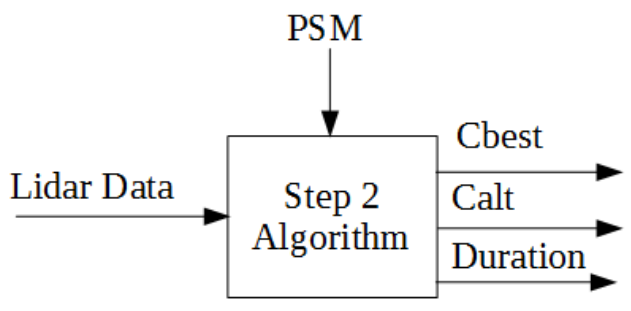

Figure 2. Step 2 block diagram

Vegetation Block Definition: Algorithm outline:

1. Sample the row in $10 \mathrm{~cm}$ vegetation slices.

2. For each slice, count the number of LiDAR beam interceptions in each section.

3. Detect missing vegetation slices.

4. Group adjacent missing vegetation slices.

5. Form vegetation blocks and proceed as in 2 for counting LiDAR beam interceptions.

6. Group adjacent vegetation blocks with similar characteristics. 
7. Search for $\mathrm{C}_{\text {best }}$ and $\mathrm{C}_{\text {alt }}$ for each vegetation block.

8. Convert lengths of blocks to durations.

\subsection{Step 3: The Timed Automata Model used}

A model based on a network of Timed Automata was developed. It represents the behaviour of each nozzle (nozzles automata), the selection of the command for the next block (anticipation automaton), the displacement of the sprayer inside the row from block to block (displacement automaton), the starting of spray in the row (row start automaton), and the opening and closing of each nozzle according to chosen spraying configuration command (control nozzles automaton).

In the following, each automaton is explained and the synchronization between the automata is described by the block diagram of Figure 3 .

Row Start automaton: This automaton manages the startup phase. It sends two command events "startAnticip" (to select a command for the first block) and "startBlock" (to start spraying at the first block)."

Anticipation automaton: The role of this automaton is to select a command for the next block. The two possible commands for each vegetation block are $\mathrm{C}_{\text {best }}$ and $\mathrm{C}_{\text {alt }}$, as resulting from applying step2 of AMPS to LiDAR data. After the choice of a command, the anticipation automaton sends the event "finishAnticip".

Displacement automaton: It represents the actual displacement of the sprayer. At first, it receives the start synchronization event "startBlock" from the Row Start automaton. Then, it sends the "newBlock" event to the Control Nozzles automaton at the end of each vegetation block. At the end of a row, it sends the "endRow" event to the Control Nozzles automaton. The time the sprayer requires to spray a whole block is calculated a priori during step 2, from the length of the block and the sprayer speed.

Control Nozzles automaton: The role of this automaton is to control the nozzles. When it receives a synchronization event "finishAnticip" from the Anticipation automaton, it calls the "control" function. This function determines, from the selected command, which nozzles must be opened and which ones must be closed for the next block. In order to secure the quantity of product sprayed in each block, the nozzles selected for opening are opened 0.2s before the beginning of the block, and conversely they are closed $0.2 \mathrm{~s}$ after its end. Control Nozzles automaton sends the "onNozzle [i]" event to open a nozzlei and "offNozzle [i]" to close it.

Nozzles automata: Each of these automaton has four states: OFF (initial state), ON, TransitionOfOpen and TransitionOfClose. It receives the synchronization events "onNozzle" and "offNozzle" to pass from a state to another. The nozzles response time (for opening or closing) is supposed to be a fixed and known value (200ms in our example sprayer). In addition, this automaton counts the quantity of sprayed product using the derivative function cost'. This function, implemented in UppAal-CORA, represents the flow of product at any time. The higher the flow, the higher the cost will be at the end.

The synchronization between automata is described by the following block diagram. In UppAal, time constraints are expressed with integer values. In the model used in this paper, 1 clock unit represents $0.1 \mathrm{~s}$.

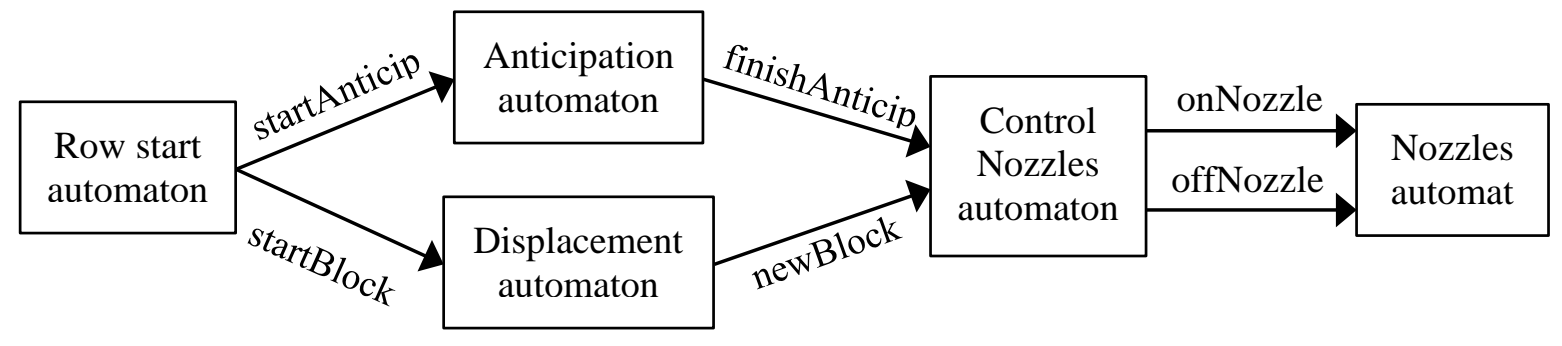

Figure 3. Step 3 block diagram 


\subsection{Step3: Computing the Control Sequence using UppAal-Cora}

The model described above has been designed in order to be adaptable to a wide range of sprayer types. It has been made with the UppAal-CORA tool. In the step 3 of the AMPS method, UppAalCORA model-checking is used to compute and verify the control sequence that matches the behavioral constraints. Model-checking is done by checking properties on a model. Here, the model checking is used to verify the following property: is there a control sequence that allows the sprayer to reach the end of the row (while applying the control constraints specified in the model)? UppAal-CORA checks this property and provides a trace with the lowest cost if such a control sequence exists: this is the optimal control sequence. Because the derivate cost' of the cost function specified in the model is the flow rate, the calculated optimal cost is the minimal amount of product necessary to spray the considered row.

\section{Results \& Discussion}

In this section, we will assess the feasibility of applying the AMPS method for real vine LiDAR data. We evaluate precision spraying possibilities while taking into account the dynamics of the sprayer. The calculated control sequence will be compared to classical spraying without automation.

\subsection{LiDAR data}

As a benchmark, we consider one row of a vine plot in South of France planted with Chardonnay which was scanned with a 2D LiDAR such as presented in (Bastianelli et al, 2017).

A part of the vegetation profile of the studied row is presented in Figure 4. The number of LiDAR interceptions is rendered as grey level in this figure. This profile shows a lot of missing vegetation. For example, the vegetation block framed by $\mathrm{x}=[5.8 \mathrm{~m}: 7.2 \mathrm{~m}]$ and $\mathrm{y}=[0.5 \mathrm{~m}: 1.1 \mathrm{~m}]$ is a missing vegetation block (Yellow boxes in Figure 4). There are also many cases where the value 0 must be assigned to the $\mathrm{H}$ section (area above 1.2 shown with orange colour).

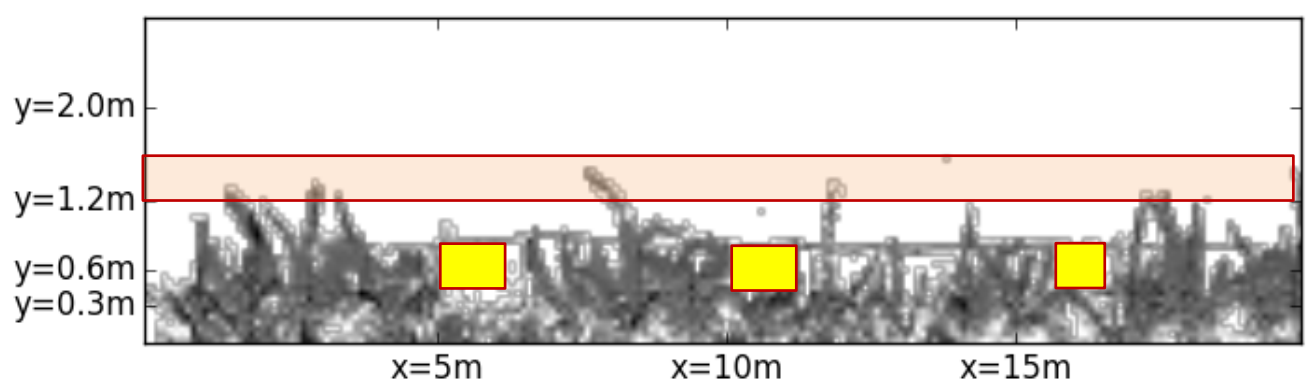

Figure 4. Extract of studied vegetation profile 


\subsection{Computation of spraying configuration commands map}

As said previously, the AMPS step 2 consists in the processing of the LiDAR data to obtain $\mathrm{C}_{\text {best }}$, $\mathrm{C}_{\text {alt }}$ and the duration of each vegetation block for the studied row. The studied row is 112 meters long and the sprayer is supposed to move at $1.4 \mathrm{~m}_{\mathrm{s}} \mathrm{s}^{-1}$. Applying the step 2 algorithm, a sequence of 161 blocks was computed, with 10 blocks that have no vegetation. The length of a block is expressed as a function of the time needed to spray it. The smallest duration of a block is $0.4 \mathrm{~s}$ and the longest is 1.4s. $71 \%$ of the blocks have the smallest duration. The duration of $8 \%$ of the blocks is greater than $1 \mathrm{~s}$. The obtained map of control set points $\mathrm{C}_{\text {best }}$ and $\mathrm{C}_{\text {alt }}$ is given in Figure 5. To each type of control command a colour is assigned: LH (bleu), CH (red), HH (green), LH\&CH (magenta), LH\&HH (cyan), CH\&HH (yellow) and -- (white) for hands all switched off. The command width is the duration of the block.
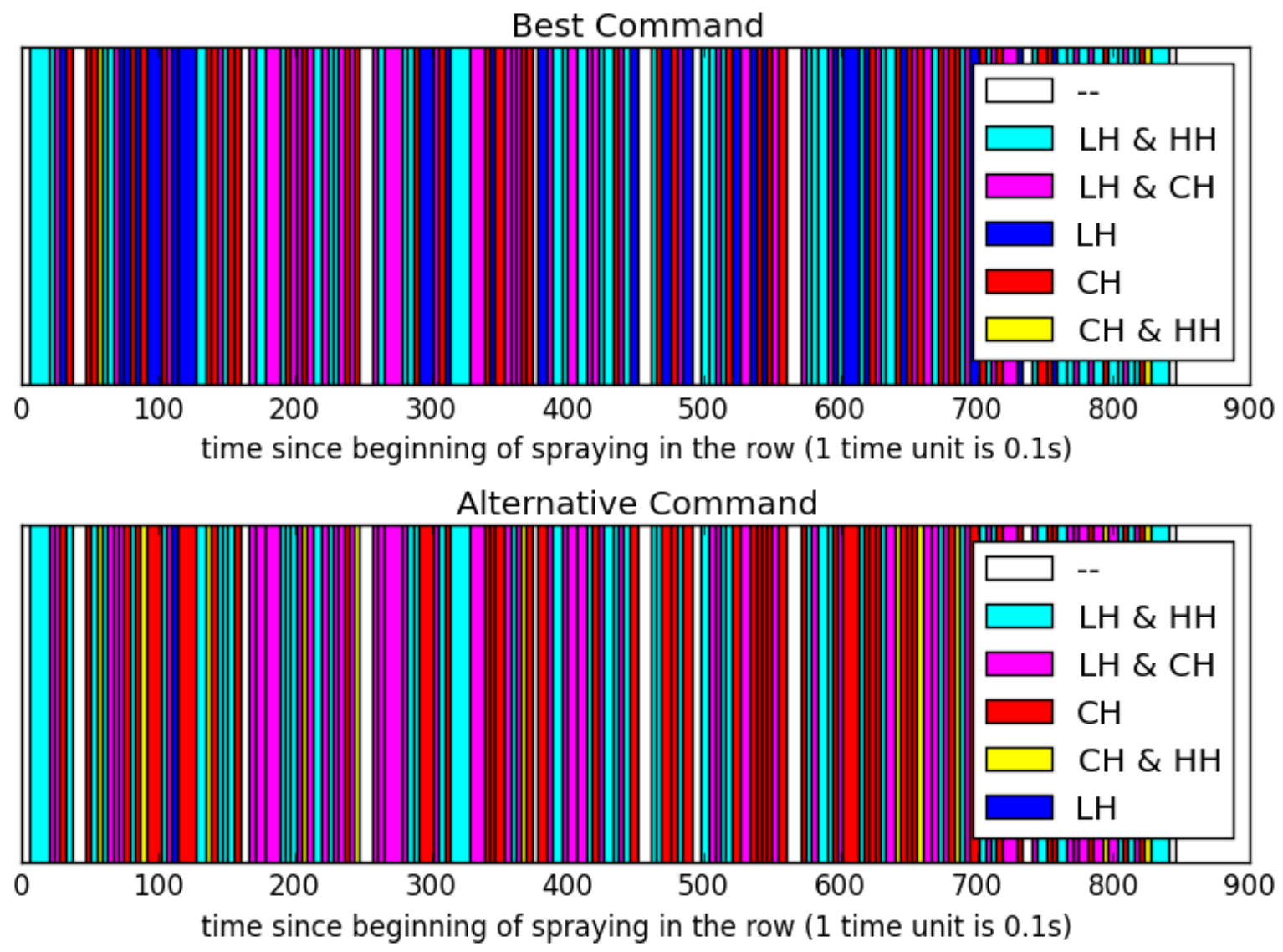

Figure 5. Output from step 2: Best and alternative commands in the studied row

From step 2 output, the optimal control sequence choosing between $\mathrm{C}_{\text {best }}$ and $\mathrm{C}_{\mathrm{alt}}$ and minimizing the spraying liquid quantity must be computed.

\subsection{Computation of optimal control sequence}

Using UppAal-CORA, queries provide for checking the existence of a control sequence matching behavior constraints. When at least one such control sequence exists, UppAal-CORA can provide the one with the lowest cost: optimal command. Figure 6 shows the optimal control sequence with the lowest cost provided by UppAal-CORA. In this sequence, $\mathrm{CH}$ (red color) is the most frequent command. It appears in 61 vegetation blocks. The LH\&CH command (magenta) appears in 48 vegetation blocks. The CH\&HH (yellow) command is present only twice.

It appears in the optimal solution that one hand is sufficient to spray $43 \%$ of the blocks, while a classical sprayer would always use two hands. So, the AMPS method succeeds to apply a previously determined map of pesticide dose and sprayer configuration commands considering the control

Rim Saddem, Olivier Naud, Paul Cazenave, Karen Godary Dejean, Didier Crestani: Precision spraying: from map to sprayer control using model-checking 
dynamics of a sprayer. Moreover, the method reduces the quantities of chemicals sprayed while maintaining yield and quality. This result is more realistic than a static map with a unique spraying configuration for each block. Often, in precision spraying, response time of nozzles is not taken into account. The AMPS method provides a detailed analysis of dose reduction possibilities. These are discussed in the sequel.

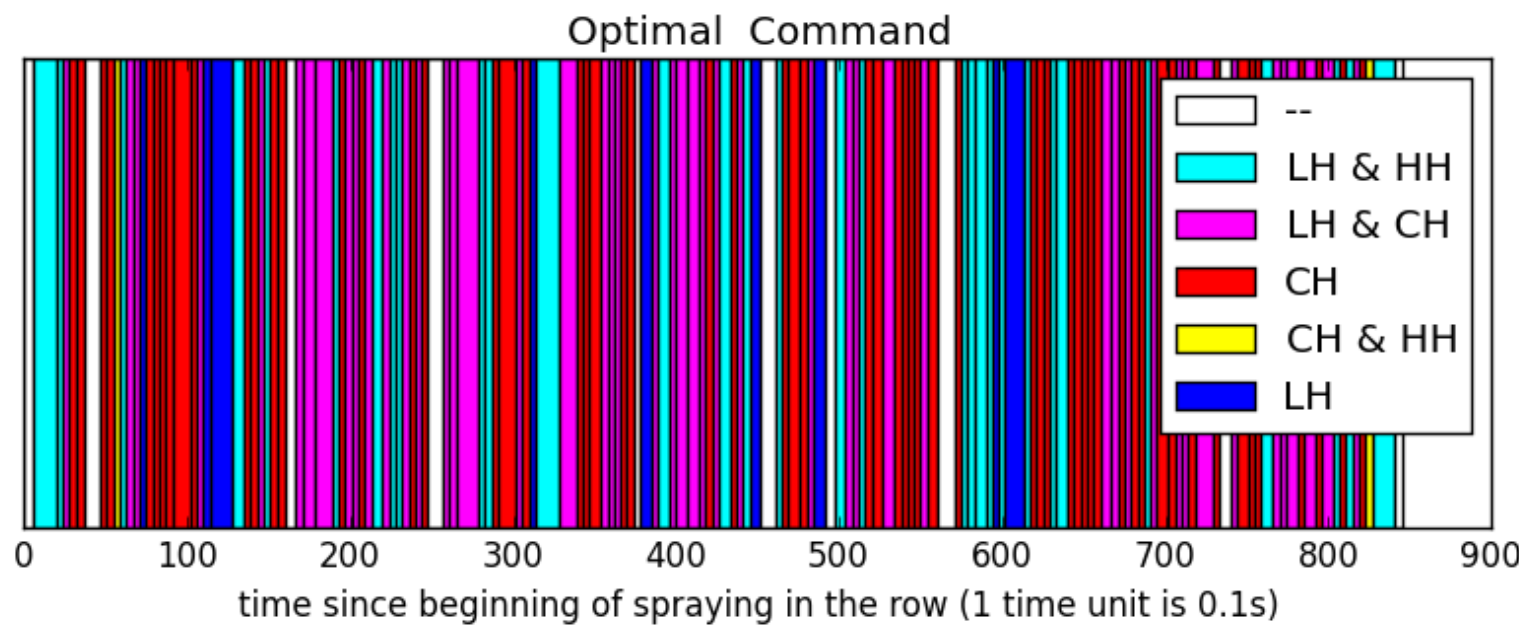

Figure 6. Computed Optimal Sequence

We will now compare this optimal command and two other possible ones with the classical spraying method. Table 2 shows a comparative table of savings according to selected sequence of commands. A classical sprayer uses the LH\&HH hands configuration, which would result for the 161 blocks in spraying the nominal flow rate for 84,5 seconds (index A). Considering now the possibility to separately and dynamically command nozzles, we could first consider the theoretical best control sequence, applying a $\mathrm{C}_{\text {best }}$ mapping without taking into account the sprayer dynamics (supposing then instantaneous response). This sequence results in saving $28 \%$ compared to the nominal spray amount (index B). But the dynamics of the sprayer is a mandatory constraint, and have to be considered. Then, the real results of applying only $\mathrm{C}_{\text {best }}$ commands leads to save only $14 \%$ of product (index $\mathrm{C}$ ), while applying a mix of $\mathrm{C}_{\text {best }}$ and $\mathrm{C}_{\text {alt }}$ commands depending on the optimal sequence provided by UppAalCORA (index D) allows to save in average $19 \%$ of this amount while maintaining sufficient local protection. Thus, choosing a unique local ideal command for each block does not provide the optimal spraying on the whole field due to transition times in opening and closing the nozzles. This is precisely the practical problem addressed in this paper.

Table 2. Comparative table of savings according to selected commands (C\&D computed with AMPS method).

\begin{tabular}{|l|c|c|}
\hline Index & Command & Savings \\
\hline A & Classical spraying: LH \& HH command for all blocks & $0 \%$ \\
\hline B & $\begin{array}{c}\text { Theoretical best command on all blocks: Cbest on all blocks without } \\
\text { considering sprayer dynamics. }\end{array}$ & $28 \%$ \\
\hline C & Only best command on all blocks considering sprayer dynamics & $14 \%$ \\
\hline D & Optimal command considering sprayer dynamics & $19 \%$ \\
\hline
\end{tabular}

As an explanatory example, let us consider two consecutive vegetation blocks such that the first has the characteristics T-H-M-L $=(1212)$ and the second has the characteristics T-H-M-L $=(2021)$. For the first block and using the PSM function, $\mathrm{C}_{\text {best }}=\mathrm{LH} \& \mathrm{HH}$ and $\mathrm{C}_{\text {alt }}=\mathrm{LH} \& \mathrm{CH}$. For the second block, $\mathrm{C}_{\text {best }}=\mathrm{LH} \& \mathrm{CH}$ and $\mathrm{C}_{\text {alt }}=\mathrm{LH} \& H H$. To compute the optimal sequence, uppaal cora selects $\mathrm{C}_{\text {best }}$ for the first block and $\mathrm{C}_{\text {alt }}$ for the second. If $\mathrm{C}_{\text {best }}$ was applied for both blocks, then $\mathrm{CH}$ should be activated $200 \mathrm{~ms}$ before the end of the first block, and HH should be activated $200 \mathrm{~ms}$ after the start of second block, resulting in the unnecessary waste of some product. 


\section{Conclusion}

In this paper, we investigated how model-checking and timed automata can contribute to precision agriculture, for the development of command methods in order to optimise accurate spraying according to vegetation density. We have proposed the Automata Modelling for Precision Spraying (AMPS) method that assesses the feasibility of applying a dose and spraying configuration map considering the dynamics of a sprayer hand, and that computes the optimal control sequence thanks to exhaustive command state space search. We show that the AMPS method is applicable to some real vine LiDAR data. The obtained optimal control sequence indicates that reducing phytosanitary products is possible while maintaining a sufficient protection on each plant. AMPS takes into account the control dynamics of the used sprayer. If sprayer dynamics were not taken into account, then potential savings on phytosanitary products would be overestimated. The main interest of AMPS method is to estimate, before the development of a specific system, the gains that could be made with an automatic multi-spouts sprayer.

The AMPS method is based on the timed automata formalism and the UppAal-CORA modelchecking tool. The UppAal-CORA model-checking tool presents some limitations. Only reachability properties can be checked, liveness properties and deadlock property cannot be checked. In addition, the available binary has been built only for 32 architectures which is limited to 4GB of RAM. The complexity of deciding between two alternative commands for every block of a row is exponential with regards to the number of blocks. To check the reachability property on the whole vine row, some spatial decomposition has been needed. This spatial decomposition issue will be discussed in a future paper.

The AMPS method may now be integrated in a graphic tool. This tool would take as input the LiDAR data of grapevine plots and the characteristics of a sprayer and would provide as output the optimal sequence of spraying configuration commands to be applied in the row. One other perspective is to apply the method in a set of different plots with contrasting vegetation characteristics and analyse the potential of automation for reducing quantities sprayed in various vineyards.

\section{Acknowledgment}

This work has received the support of French National Research Agency under the grant number ANR-14-CE27-0004 attributed to the AdAP2E project. We also want to thank collegues involved in agricultural research project CASDAR ArchiTechDoseViti for providing the LiDAR data.

\section{References}

Alur, R., Dill, D.L., 1994. A theory of timed automata. Theoretical Computer Science, vol. 126, pp. 183235. https://doi.org/10.1016/0304-3975(94)90010-8

Bastianelli, M, de Rudnicki, V,, Codis, S, Naud, O. 2017. Two vegetation indicators from 2D ground Lidar scanner compared for predicting spraying deposits on grapevine. Communication accepted for EFITA WCCA 2017 int. conf., July 2-6, Montpellier.

Behrmann, G., Larsen, K.G., Rasmussen, J.I., 2005. Optimal Scheduling Using Priced Timed Automata, ACM SIGMETRICS Performance Evaluation Review, vol. 32, pp. 34-40. https://doi.org/10.1145/1059816.1059823

Berk, P, Hocevar, M, Stajnko, D, Belsak, A, 2016, Development of alternative plant protection product application techniques in orchards, based on measurement sensing systems: A review, Computers and Electronics in Agriculture, vol. 124, pp. 273-288. http://dx.doi.org/10.1016/j.compag.2016.04.018

Gil, E., Escolà, A., Rosell, J.R., Planas, S., Val, L., 2007.Variable rate application of plant protection products in vineyard using ultrasonic sensors.Crop Protection 26, 1287-1297. https://doi.org/10.1016/j.envsoft.2011.08.005

Hall, A., Louis, J., Lamb, D., 2003. Characterising and mapping vineyard canopy using high-spatial-resolution aerial multispectral images. Computers \& Geosciences 29, 813-822. https://doi.org/10.1016/S0098$\underline{3004(03) 00082-7}$ 
Hélias, A., Guerrin, F., Steyer, J.-P., 2008. Using timed automata and model-checking to simulate material flow in agricultural production systems-Application to animal waste management. Computers and Electronics in Agriculture 63, 183-192. doi:10.1016/j.compag.2008.02.008. https://doi.org/10.1016/j.compag.2008.02.008

Largouët, C., Cordier, M.-O., Bozec, Y.-M., Zhao, Y., Fontenelle, G., 2012. Use of timed automata and modelchecking to explore scenarios on ecosystem models. Environmental Modelling \& Software 30, 123138. https://doi.org/10.1016/j.envsoft.2011.08.005

Larsen, K.G., Pettersson, P., Yi, W., 1997. Uppaal in a nutshell, Int. Jal on Software Tools for Technology Transfer (STTT), vol. 1, pp. 134-152. https://doi.org/10.1007/s100090050010

Naud, O., Verges, A., Hebrard, O., Codis, S., Douzals, J. P., Ruelle, B, 2014. Comparative assessment of agroenvironmental performance of vineyard sprayers using a physical full scale model of a vineyard row, AgEng 2014, Zürich.

Pai, N., Salyani, M., Sweeb, R.D., 2009. Regulating airflow of orchard airblast sprayer based on tree foliage density. Transactions of the ASABE 52, 1423-1428. https://doi.org/10.13031/2013.29122

Rosell, J.R., Llorens, J., Sanz, R., Arnó, J., Ribes-Dasi, M., Masip, J., Escolà, A., Camp, F., Solanelles, F., Gràcia, F., Gil, E., Val, L., Planas, S., Palacín, J., 2009. Obtaining the three-dimensional structure of tree orchards from remote 2D terrestrial LIDAR scanning. Agricultural and Forest Meteorology 149, 15051515. https://doi.org/10.1016/j.agrformet.2009.04.008

Scopélitis, J., Andréfouët, S., Largouët, C., 2007. Modelling coral reef habitat trajectories: Evaluation of an integrated timed automata and remote sensing approach. Ecological Modelling 205, 5980. https://doi.org/10.1016/j.ecolmodel.2007.02.011 